\title{
Stereotactic Body Radiation Therapy (SBRT) for prostate cancer: Preliminary results of toxicity
}

\author{
Asmâa Naim * 1,3, Safae Mansouri ${ }^{*}$, Kamal Saidi ${ }^{3}$, Abdeljalil Heddat ${ }^{1,4}$, Younes Elhoury ${ }^{1,4}$, \\ Redouane Rabii ${ }^{1,4}$ \\ ${ }^{1}$ Mohammed VI University of Health Sciences (UM6SS), Morocco; \\ ${ }^{2}$ Oncology Center of Meknes, Morocco; \\ ${ }^{3}$ Department of Radiotherapy, Casablanca Cancer Center, International hospital Cheikh Khalifa, Casablanca, Morocco; \\ ${ }^{4}$ Department of Urology, International Hospital Cheikh Khalifa, Casablanca, Morocco. \\ * Both Authors had participated equally to this manuscript.
}

Submitted 18 April 2021; Accepted 27 April 2021

To the Editor,

Prostate cancer is the second most common cancer in men in Morocco after lung cancer. External radiotherapy (RTE) is a curative therapeutic option for localized prostate cancer, However the conventional RTE remains a long treatment (78 weeks, 5 days a week) which is demanding for patients and make difficult to manage the waiting lists.

The development of imaging and irradiation techniques over the last decades has allowed a high precision in the delivery of the dose to the target organ and a better protection of the organs at risk (OAR), which has encouraged the hypo fractionated irradiation of localized prostate cancer, especially after the results of radiobiology studies that suggested a low report $\alpha / \beta$ for the prostate.

Therefore, several trials have attempted to prove the efficacy and tolerance of hypo fractionated regimens ranging from moderate hypofractionation (20 sessions) to extreme hypofractionation (4-7sessions) called also Stereotactic Body Radiotherapy (SBRT). In fact, it has been demonstrated the same toxicity profile as normo-fractionated (1-3) as well as non-inferiority for tumor control $(6,8-10)$.

Advanced technology in radiotherapy like SBRT is still difficult to access in incoming countries, although we have the opportunity to practice this technique in our institution.

The aim of our paper is to share our experience and to present our preliminary results in term of toxicity after SBRT of low and intermediate risk prostate cancer.

From January 2017 to December 2018, we treated patients with stereotactic radiotherapy at a total dose of 36.25 Gy in 5 sessions of $7.25 \mathrm{~Gy}$. Prostatic re-irradiation was excluded.

\section{Methods}

All our cases were discussed in Multidisciplinary Consultation Meeting including urologist, oncologist, radiotherapist, radiologist and pathologist and decision of stereotactic radiotherapy was validated. After patient consenting, the team of urology procced to transrectal placement of electromagnetic markers Calypso ${ }^{\circledR}$ transponders to follow the prostate during radiotherapy treatment. In fact, the transponders allow a real time tracking of the target as well as a perfect repositioning of the prostate in intrafraction.

The placement of the transponders proceeds as fellow: administration of premedication as prescribed by the anesthetist, lithotomy positioning of the patient, transrectal ultrasound in the transverse and sagittal planes, determination of the position by means of three-dimensional probe tracking, intra-prostatic implantation of three transponders ( 2 at the base and 1 at the apex).

After 7-10 days after transponders have been inserted, a simulation scan is performed as follow: rectal and bladder preparation, dorsal decubitus with means of restraint (knee block/foot block), sub-millimetric scan sections.

The radiotherapist proceeds to the delineation of the target volumes and organs at risk specially the urethra.

Target volume received a dose of 3625 cGy in 5 fractions, considering $\alpha / \beta=1.5$ and a Biological Dose Equivalent (BED) of 286 Gy.

The clinical target volume was covered by $100 \%$ of the prescribed doses, all organs at risk were delineated and doses constraints were met for all according to SFRO recommendations and the TG101 report of the medical physics $(4,5)$.

Then the medical physician starts to plan a treatment and finally the best proposition of treatment is validated by the radiotherapist before to initiate the treatment.

All patients were treated with the latest generation linear accelerator (True Beam STX). Calypso was used as tracking system: the electromagnetic antenna of its optical system allowed real time tracking of the target.

No conflict of interest declared.

Archivio Italiano di Urologia e Andrologia 2021; 93, 3 


\section{Biological control}

Increasing doses per fraction may improve the carcinology outcome. In fact, the meta-analysis of Zaorsky has shown that when the Biological Equivalent Dose is between 140 Gy to $200 \mathrm{~Gy}$ we have a better tumor control for prostate cancer. It has been also reported that SBRT improved by $12 \%$ biological progression free survival (bPFS) $(6,7)$. Phase I-III trials proved the non-inferiority of SBRT compared to the standard regimen in terms of tumor control and acute and delayed toxicity 8-10]. SBRT is still an option as an alternative for the treatment of low-risk prostate cancer and may be considered for intermediate risks in multi-center trials. In our study, we achieved BED > 200 Gy but it is still too early to assess biological control. At 2 years, nine patients were in biological control, two patients died by other diseases: lung cancer and pulmonary embolism.

\section{Acute toxicity}

Acute toxicity considered any complication occurring within 3 months of the end of irradiation. Genitourinary (GU) acute complications were cystitis grade 1 and 2 (with a frequency of 25\%); no acute grade 3 GU toxicity was seen.

Gastrointestinal (GI) acute complications were proctitis grade 1 and 2 (with a frequency of 8.4\%); no grade 3 GI toxicity was seen. Our results are similar to the literature $(11,12)$.

\section{Late toxicity}

In our preliminary results, no late GI toxicity was detected but we had two cases of urethral stenosis.

In Jackson's meta-analysis, concerning 6000 patients treated in 38 prospective studies, with two phase III randomized trials and a median follow-up of at least 5 years, late toxicity Grade $\geq 3$ GU was 2.2\% and GI was 0.8\% (12).

Pan et al. reported similar late GU and GI toxicity but they also observed a significantly higher risk of urinary fistula at 2 years after SBRT ( $1 \%$ vs. $0.1 \%$; $\mathrm{p}=0.009$ ) (13).

Due to the heterogeneity of risk groups, doses and techniques used in different trials, long-term results of further phase III trials are underway to confirm the current data.

Trials are open to evaluate SBRT in high-risk prostate cancer (SPARC trial /PACE C) and others to evaluate fractionation and optimal dose: (PATRIOT) $(14,15)$. Meanwhile, our preliminary results are extremely encouraging in terms of toxicity and local control but we need longer follow up and a larger sample to draw relevant conclusions for this innovative treatment of localized prostate cancer.

\section{REFERENCES}

1. Katz AJ, Kang J. Quality of life and toxicity after SBRT for organ-confined prostate cancer, a 7-year study. Front Oncol. $2014 ; 4: 301$.

2. Widmark A, Gunnlaugsson A, Beckman L, et al. Ultrahypofractionation for prostate cancer: Outcome from the Scandinavian phase 3 HYPORT-PC trial. Lancet 2019; 394(10196):385-395.

3. Van As NJ, Brand D, Tree A, et al. PACE: Analysis of acute toxicity in PACE-B, an international phase III randomized controlled trial comparing stereotactic body radiotherapy (SBRT) to conventionally fractionated or moderately hypofractionated external beam radiotherapy (CFMHRT) for localized prostate cancer (LPCa). J Clin Oncol. 2019; 37(Suppl 7):1-1.

4. Noël G, Antoni D, Barillot I, et al. Délinéation des organes à risque et contraintes dosimétriques. Cancer/Radiothérapie. 2016; 20 Suppl:S3660.

5. Benedict SH, Yenice KM, Followill D, et al. Stereotactic body radiation therapy: The report of TG101. Med Phys. 2010; 37:4078-101.

6. Hoffman KE, Voong KR, Levy LB, et al. Randomized trial of hypofractionated, dose-escalated, intensity-modulated radiation therapy (IMRT) versus conventionally fractionated IMRT for localized prostate cancer. J Clin Oncol. 2018; 36:2943-2949.

7. Zaorsky NG, Palmer JD, Hurwitz MD, et al. What is the ideal radiotherapy dose to treat prostate cancer? A meta-analysis of biologically equivalent dose escalation Radiother Oncol. 2015; 115:295-300

8. Aluwini S, Pos F, Schimmel E, et al. Hypo fractionated versus conventionally fractionated radiotherapy for patients with prostate cancer (HYPRO): Late toxicity results from a randomized, non-inferiority, phase 3 trial. Lancet Oncol. 2016; 17:464-474

9. Dearnaley D, Syndikus I, Mossop H, et al. Conventional versus hypo fractionated high-dose intensity- modulated radiotherapy for prostate cancer: 5- year outcomes of the randomized, non- inferiority, phase 3 CHHiP trial. Lancet Oncol. 2016; 17:1047-1060.

10. Hoffman KE, Skinner H, Pugh TJ, et al. Patient reported urinary, bowel, and sexual function after hypofractionated intensity-modulated radiation therapy for prostate cancer: Results from a randomized trial. J Clin Oncol. 2018; 41:558-567.

11. Madsen BL, Hsi RA, Pham HT, et al. Stereotactic hypofractionated accurate radiotherapy of the prostate (SHARP), 33.5 gy in five fractions for localized disease: first clinical trial results. Int J Radiat Oncol Biol Phys. 2007; 67:1099-105.

12. Jackson WC, Silva J, Hartman HE, et al. Stereotactic body radiation therapy for localized prostate cancer: a systematic review and metaanalysis of over 6,000 patients treated on prospective studies. Int J Radiat Oncol Biol Phys. 2019; 104:778-789.

13. Pan HY, Jiang J, Hoffman KE, et al. Comparative toxicities and cost of intensity-modulated radiotherapy, proton radiation, and stereotactic body radiotherapy among younger men with prostate cancer. ,J Clin Oncol. 2018; 36:1823-1830.

14. Morrison K, Tree A, Khoo V, et al. The PACE trial: International randomised study of laparoscopic prostatectomy vs. stereotactic body radio-

Archivio Italiano di Urologia e Andrologia 2021; 93, 3 
therapy (SBRT) and standard radiotherapy vs. SBRT for early stage organ- confined prostate cancer. J Clin Oncol. 2018; 36(Suppl 6):TPS153TPS153.

15. Quon HC, Ong A, Cheung P, et al. Once-weekly versus every-other-day stereotactic body radiotherapy in patients with prostate cancer (PATRIOT): a phase 2 randomized trial. Radiother Oncol. 2018; 127:206-212.

\section{Correspondence}

Asmâa Naim, MD (Correspondent Author)

doc.a.naim@gmail.com

Hôpital International Cheikh Khalifa, Casablanca (Morocco)

Safae Mansouri, MD

m-safae@hotmail.fr

Oncology Center of Meknes, Meknes, Morocco

Kamal Saidi, Medical physicist

Department of Radiotherapy, Casablanca Cancer Center

Hôpital International Cheikh Khalifa, Casablanca (Morocco)

Abdeljalil Heddat, MD

Younes Elhour, MD

Redouane Rabii, MD

Department of Urology, Hôpital International Cheikh Khalifa, Casablanca

(Morocco) 